\title{
EL DISEÑO EMOGIONAL COMO \\ BASE PARA LA MEJORA DE EXPERIENCIAS
}

\section{EMOTIONAL DESIGN AS A GORNERSTONE FOR EMOTIONAL ENHANGEMENT}

\author{
Adriana Guadalupe Leija Macías \\ Trabajadora independiente - México
}

Diseñadora Industrial de profesión. Competencias y habilidades desarrolladas como fotógrafa profesional y dedicada al giro. Co-propietaria y fundadora de la empresa Enfocart Photography, cuyo modelo de negocio es la fotografía profesional y toma de video en ambientes y entornos sociales, así como fotografía de producto. Alto interés y sentido de aprendizaje continuo por la ingeniería detrás del diseño. Y como principal pasatiempo, un gusto particular por la naturaleza y los seres vivos.

adriana.leija12@outlook.com

\section{Sofía Alejandra Luna Rodríguez \\ Universidad Autónoma Nuevo León - México}

Diseñadora Industrial egresada de la Facultad de Arquitectura de la Universidad Autónoma Nuevo León (UANL), y donde tuvo a su cargo la coordinación administrativa del Centro de Formación, Capacitación y Actualización Docente, así como la Coordinación de Intercambio Académico. Cuenta con una Maestría en Artes, con acentuación en Educación en el Arte, por la Facultad de Artes Visuales de la UANL. Maestra fundadora de la Licenciatura en Diseño Industrial del Centro de Estudios Superiores de Diseño de Monterrey (CEDIM). Con un Doctorado por el programa DADU, con líneas de investigación sobre educación y teoría en el diseño. Cuenta con publicaciones y ponencias a nivel nacional e internacional. Actualmente enfocada a la docencia e investigación académica dentro de la UANL. Candidato a Investigador Nacional (SNI). Fundadora del Grupo de Investigación en Diseño DAMATEUR.

sofia.lunard@uanl.edu.mx

Fecha de recepción: 24 de agosto, 2018 / Aceptación: 20 de octubre, 2018. 


\section{Resumen}

En México un beneficio que varias empresas aportan a los trabajadores es el seguro médico por parte de los hospitales públicos. Al ser uno de los lugares mayormente concurridos, en los últimos años se ha notado una sobrepoblación en los hospitales y clínicas de esta institución. En ocasiones, es necesario que la persona internada tenga a un familiar cercano el cual lo ayudará a realizar actividades dentro de la estancia en el hospital, este familiar pasa a ser un cuidador que la mayoría del tiempo se encuentra sentado, siendo este el principal usuario para el desarrollo de un producto que ayude a combatir la fatiga de la mano con el diseño emocional y el diseño de experiencia, los cuales tienen como objetivo generar sensaciones y sentimientos en los productos o servicios. Por consiguiente, se pudieron identificar deficiencias muy puntuales, por ejemplo, mala higiene en la infraestructura física e instalaciones, equipo y mobiliario dañado, carente vigilancia y en general condiciones críticas para la estancia de los usuarios. De manera que, se realizó una búsqueda para mejorar este tipo de necesidades partiendo del Diseño Industrial.

\section{Palabras clave}

Diseño emocional, experiencia, hospital público, salud pública, mobiliario.

\section{Abstract}

In Mexico, a benefit that several companies bring to workers is health insurance in public hospitals. However, as public hospitals have become one of the most popular places in recent years, overpopulation in these institutions has been noticed. In certain occasions, patients need to have a close relative to help them perform activities within the hospital. These relatives become caregivers who remain seated most of the time. These caretakers inspired the development of a product to fight hand fatigue by using emotional and experience design. Very specific deficiencies were identified: poor hygiene in physical infrastructure and facilities, damaged equipment and furniture, lack of surveillance and critical conditions for the patient's companion stay. As a result, a search was carried out to improve this type of needs based on Industrial Design.

\section{Keywords}

Emotional design, experience, public hospital, public health, furniture. 


\section{Introducción}

En el actual artículo se aborda una temática bastante particular de buscar bienestar y experiencias confortables al ser humano. Dicho lo anterior, es común escuchar que esto en muchas ocasiones no se cumple cuando a hospitales públicos se refiere. En México, por lo general, las quejas son frecuentes y los comentarios en su mayoría negativos hacia las instalaciones físicas de los hospitales públicos. Sin embargo, en el presente, no se habla de los pacientes, sino más bien, de los usuarios indirectos, es decir, familiares, amigos o personas (a quienes dentro de este artículo llamaremos cuidadores) a cargo de dichos pacientes durante su estadía de recuperación física en la clínica u hospital en cuestión, quienes pasan por desgaste físico, emocional e impotencia, así como momentos de malestar en el cuerpo, por mencionar algunas de las situaciones vividas por las personas quienes cuidan de un ser querido que atraviesa por una estadía en algún hospital. Partiendo de esta premisa, surge la necesidad de tener como objeto de estudio el diseño emocional y el diseño de experiencias para lograr una experiencia más satisfactoria y plena para los cuidadores.

\section{Desarrollo}

\section{Metodología a utilizar}

La investigación exploratoria tiene como objetivo explicar el problema respondiendo al porqué y las condiciones en que ocurre. Asimismo, se utiliza cuando no se ha abordado una solución o no se ha hecho suficiente estudio, sin embargo, es también apropiada en las primeras etapas del proceso durante la toma de decisiones. A su vez, se realiza una investigación descriptiva, que responde las preguntas de la problemática, de tal manera que va de la mano con la exploratoria dando como resultado un diseño de investigación planeado y estructurado.

Por consiguiente, las herramientas a utilizar en el área del diseño emocional, y en el diseño de experiencias, son el análisis semiótico del producto, SCAMPER, investigación de campo, observación, y una de las más importantes, la ingeniería Kansei. Con la finalidad de desarrollar una estrategia y crear el mensaje que se desea transmitir al usuario, de modo que, se deben considerar tres aspectos: las herramientas y metodologías que van ligadas al uso personal, tipo de relación y el tiempo de duración.

En el blog Diseño de Experiencias (2009), se menciona lo declarado por Nagamachi en 1972 sobre la ingeniería Kansei donde se considera al usuario como un eje fundamental en el desarrollo de productos. Con este se refuerza la idea que se requiere tanto de la función como de la emoción para tener como resultado la experiencia de uso y cuyo propósito principal es "diseñar productos y servicios que van más allá de satisfacer nuestras necesidades. Busca diseñar experiencias de uso y producir a través de los productos que diseñamos, sensaciones y experiencias placenteras" (p. 65).

\section{Hospitalización}

Se entiende como hospitalización al servicio en donde se interna a los pacientes mediante previa autorización del médico tratante para poder establecer un diagnóstico, recibir tratamiento y asistencia médica, y dar seguimiento a la evolución del padecimiento (Instituto de Seguridad Social del Estado de México y Municipios, 2017).

\section{El entorno de los cuidadores en hospitales públicos}

Siendo una institución pública, los recursos que se tienen son utilizados principalmente en cubrir el sueldo a sus trabajadores y pensionados, el resto va hacia sus pacientes, materiales, entre otros. Por lo tanto, estas instituciones, invierten ese poco dinero que les queda en otros aspectos, dejando en segundo o incluso en tercer plano el cuidado y mantenimiento de sus instalaciones. 
Los usuarios de hospitales públicos en numerosas ocasiones se ven forzados a esperar horas para ser atendidos ante la saturación de servicios, causando malestares y preocupaciones puesto que pasan tiempo prolongado en salas de espera y en los espacios reducidos donde se encuentra internado el paciente. En ocasiones, el paciente no se puede quedar solo durante el tiempo que está hospitalizado, sin embargo, para los cuidadores se tiene un espacio sumamente reducido, en donde solo cabe una silla convencional y el entorno que lo rodea no es amigable para estar sentado por un tiempo prolongado (Agencia Reforma, 2010). De modo que, los cuidadores necesitan tener un ambiente más ameno y tranquilo (teniendo en cuenta el espacio que se tiene) para poder trabajar y/o llevar un proceso de recuperación lo más amigable posible, sobre todo cuando los derechohabientes se encuentran en su etapa de recuperación y sus familiares están todo el tiempo a su cuidado.

\section{Propósito del cuidador en un hospital}

El usuario directo es aquel que es examinado médicamente 0 al que se le administra un tratamiento para ser curado. Es este el que más tiempo pasa en las habitaciones, dependiendo de su estado de salud se determinará qué tan dependiente 0 independiente puede llegar a ser. No todos los internos pasan por el mismo proceso, algunos duran días e incluso semanas, mientras que otros, pueden permanecer ahí por un par de horas.

A pesar de ser el paciente el usuario directo, cuando se está en la etapa de recuperación se pide, como parte del reglamento interno de institutos clínicos, que un familiar permanezca en todo momento al lado del paciente debido a que tienen que estar pendientes de las necesidades del paciente, asistirlo, y dar la autorización a procedimientos de emergencia. A ellos se los conoce como cuidadores. Los cuidadores, por lo general permanecen todo el tiempo sentados en una silla colocada a un costado de la cama. Según la investigación exploratoria realizada en hospitales públicos, la estadía promedio es de alrededor de 8 horas por día, lo que provoca cansancio, estrés e incomodidad. Ante esto surge la pregunta: ¿Cómo se puede generar una mejora sustancial en la experiencia del cuidador en México durante su estancia en un hospital público partiendo del diseño emocional?

\section{El diseño y las emociones}

Las emociones están enlazadas con el diseño de distintas maneras. El concepto de emoción, según la Real Academia Española (RAE) es la "alteración del ánimo intensa y pasajera, agradable o penosa, que va acompañada de cierta conmoción somática. Interés, generalmente expectante, con que se participa en algo que está ocurriendo" (2018, en línea), es decir, es una manera de entender el sentir de las personas y su manera de comportarse en respuesta al uso u obtención de un producto y/0 un servicio. De hecho, "recientes investigaciones han demostrado cómo los objetos que nos resultan atractivos funcionan mucho mejor. No nos limitamos a usar un producto, sino que establecemos una relación emocional con él" (Casillas, 2017, p. 43 ) debido a que algunos nos transmiten recuerdos mediante la activación de los sentidos, por ejemplo, ya sea por su olor o la textura, nos pueden hacer regresar en el tiempo a recordar algún momento 0 situación en específico que nosotros relacionamos a esas sensaciones que estamos percibiendo. De igual manera, el uso de algunos provoca un sentir de alegría o de enojo, de ansiedad, de tristeza, de esperanza, motivo que hace que nos sea más fácil o difícil desprendernos de ellos. Crossley sugirió que "diseñar debe crear conexiones emocionales relevantes entre ideas, productos, servicios y marcas" (Crossley, 2003, p. 109). Javier Cañada, director de diseño en The Cocktail y codirector de Cadius, comunidad de profesionales del diseño de interacción y la usabilidad, menciona que "el diseño emocional hace referencia a todos aquellos aspectos del diseño de productos que crean lazos con el usuario que ve más allá de lo racional" (Revista M\&M, 2016, p. 22). 


\section{Diseño emocional}

En el libro Emotional Design; why we love (or hate) everyday things escrito por Donald Norman (2004) nos recalca que "las cosas atractivas funcionan mejor - lo atractivo produce emociones positivas, haciendo que los procesos mentales sean más creativos, más tolerantes a las dificultades menores" (p. 93). De tal manera que, podemos determinar que invariablemente se ha sabido que el diseño puede evocar emociones y es un modo de entender el humor de la gente y su conducta. En base a esto, se puede entender que se presenta en nuestra reacción cuando vemos un objeto o probamos un servicio por primera vez. La forma en que funciona, su aspecto, color, textura e incluso por la nostalgia que causa, debido a que transmite una emoción y experiencia que determinará la relación a dicho producto. En el documento Diseño emocional y experiencia de usuario, Nella (2014) hace referencia a Steve Jobs, en donde menciona que "el diseño no es solo como se ve 0 cómo se siente, sino cómo funciona" (p. 84). Es decir, los colores, las tipografías y las jerarquías importan, pero lo más importante está dado por la experiencia de uso, y esta se da por la relación que se genera entre el usuario y el producto.

\section{Experiencia del usuario}

Se define como experiencia al hecho de haber sentido, conocido o presenciado a alguien 0 algo, así como un conocimiento de la vida adquirido por las circunstancias o situaciones vividas partiendo de que establece la Real Academia Española (2014). Este concepto ofrece una perspectiva más amplia acerca del uso y consumo de productos, la cual estará influida por expectativas y experiencias previas, por lo tanto, a nivel empresarial es necesario que el cliente genere experiencias para poder generar fidelidad (García del Arco, 2014).

Para poder llevar a cabo el proceso de diseño, y que esté basado en diseño de experiencias, se investiga y diseña tomando en cuenta las posibles respuestas a lo siguiente: ¿cómo percibe el usuario este objeto?, ¿qué hace?, ¿cuál es su actitud?, ¿qué siente?, ¿qué piensa?, ¿repetirán esta experiencia?, ¿la evitarán?, ¿cuál es su opinión de este producto después de cierto tiempo de utilizarlo?, entre otras cuestiones que salgan en el momento. La idea es poder tomar en cuenta todas las posibles respuestas para así saber si el producto o servicio a diseñar está tomando el camino que se pretende, o si está transmitiendo lo opuesto a la experiencia que se desea.

\section{Diseño sensorial}

Se puede definir como "el diseño intencionado de atributos de los productos como: forma, color, textura, etc., de manera que un usuario al percibir tales atributos del producto e interaccionar con él a través de sus sentidos, vea satisfechas sus necesidades e inclinaciones" (Bedolla, 2004, p. 127). Este busca satisfacer las necesidades humanas derivadas de la naturaleza perceptiva de los usuarios para que al momento de diseñar los productos se considere la importancia de satisfacer los requerimientos de los cinco sentidos de una persona mediante colores, formas, peso, textura, olores y sonidos.

\section{¿Qué se está haciendo actualmente?}

La Fundación IMSS, A.C. presentó el Programa Sillas-Cama, cuyo objetivo es que existan sillas que se hagan cama para los familiares de los derechohabientes en todas las Salas de Espera de Urgencias y Terapia Intensiva del Instituto Mexicano del Seguro Social (IMSS, 2017). Con base a la propuesta publicada por IMSS en 2017, se hace mención a los objetivos de esta silla-cama:

- Todas las salas de espera de urgencias y terapia Intensiva del IMSS deben contar con silla-cama para familiares de pacientes hospitalizados.

- Tendrá un beneficio para 11 mil personas a lo largo de su vida útil.

- Las sillas-cama son 100\% mexicanas con un costo de 2 mil 800 pesos. 


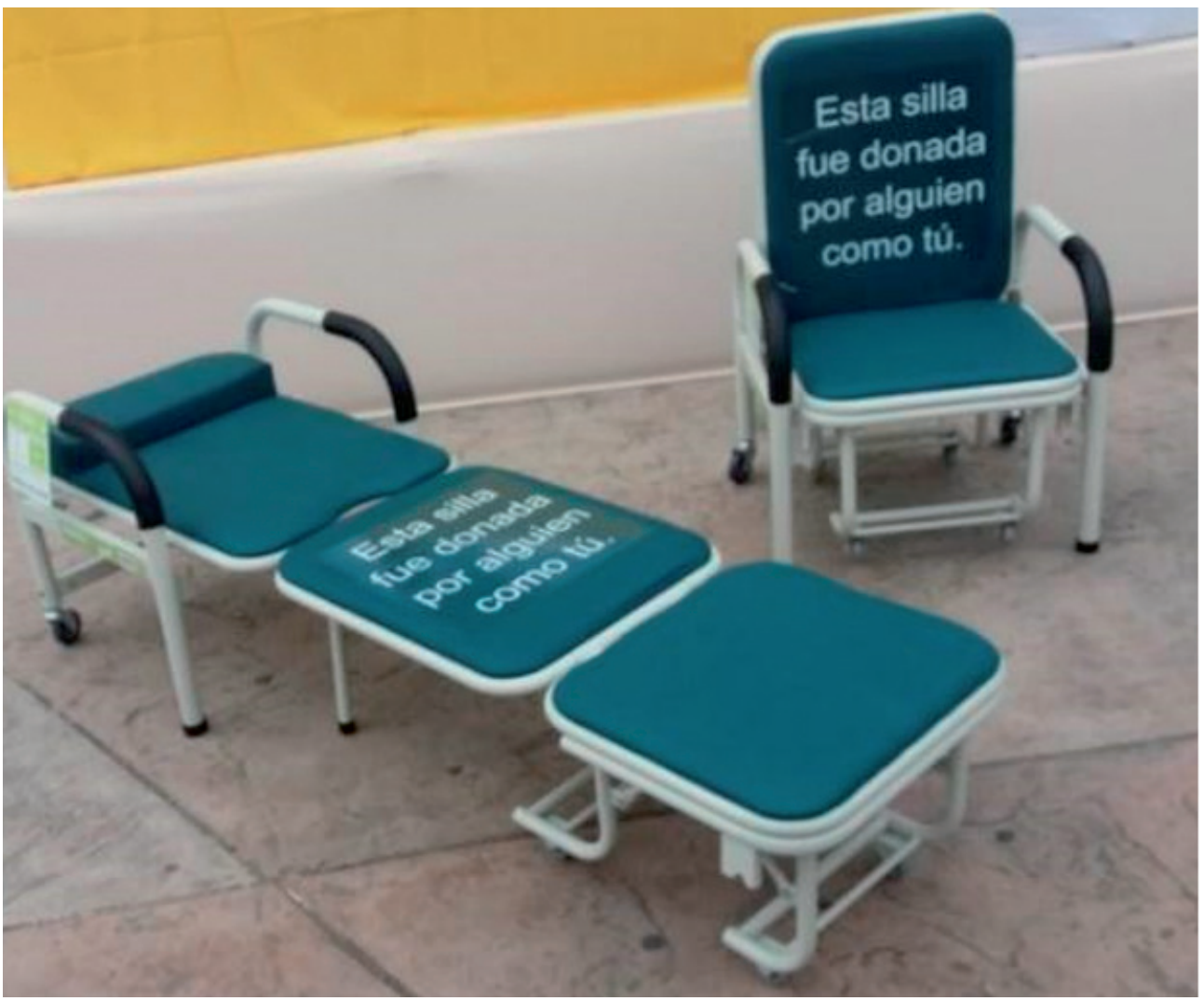

Figura 1. Análisis de similares: Silla-cama actual en las salas de espera del IMSS.

Fuente: Gobierno mexicano (2017).

Investigación de campo e interpretación de datos

Se realizó una encuesta electrónica para explorar y conocer que piensan y sienten los usuarios al estar en un cuarto de hospital, ya sea como paciente, cuidador o personal médico y de enfermería (Apéndice 1). De esa serie de preguntas se obtuvieron resultados que fueron de utilidad para generar el producto final, que se describen a continuación:
1. Los usuarios externaron que el entorno que los rodeaba los hacía sentir incómodos y tristes (Gráfica 1). 
Pregunta 1: ¿Cómo era el entorno que lo rodeaba? (Puede seleccionar más de una opción)

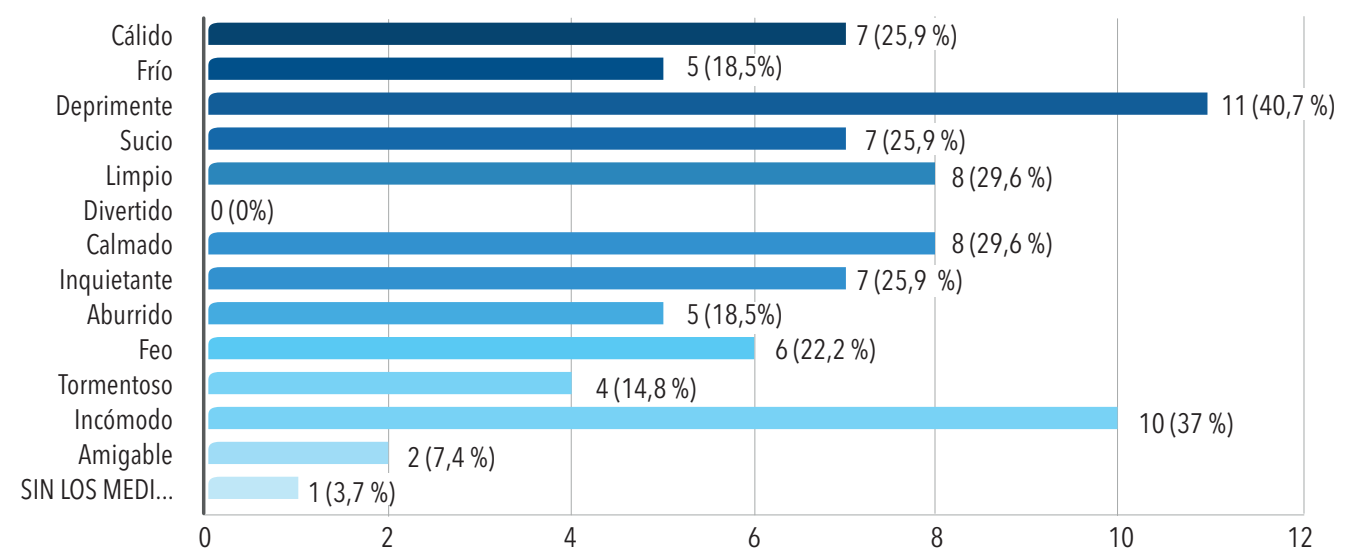

Gráfica 1. Descripción del entorno. (Leija Macías, 2018).

2. Mencionaron que hacía falta un lugar los cuidan también carecían de confort, agregando para guardar sus pertenencias, que las camas de los que eran difíciles de utilizar (Gráfica 2). pacientes no son cómodas y las sillas para quienes

Pregunta 2: Si tuviera la oportunidad de modificar algo dentro de los cuartos de recuperación, ¿qué sería? (Puede seleccionar diversas opciones).

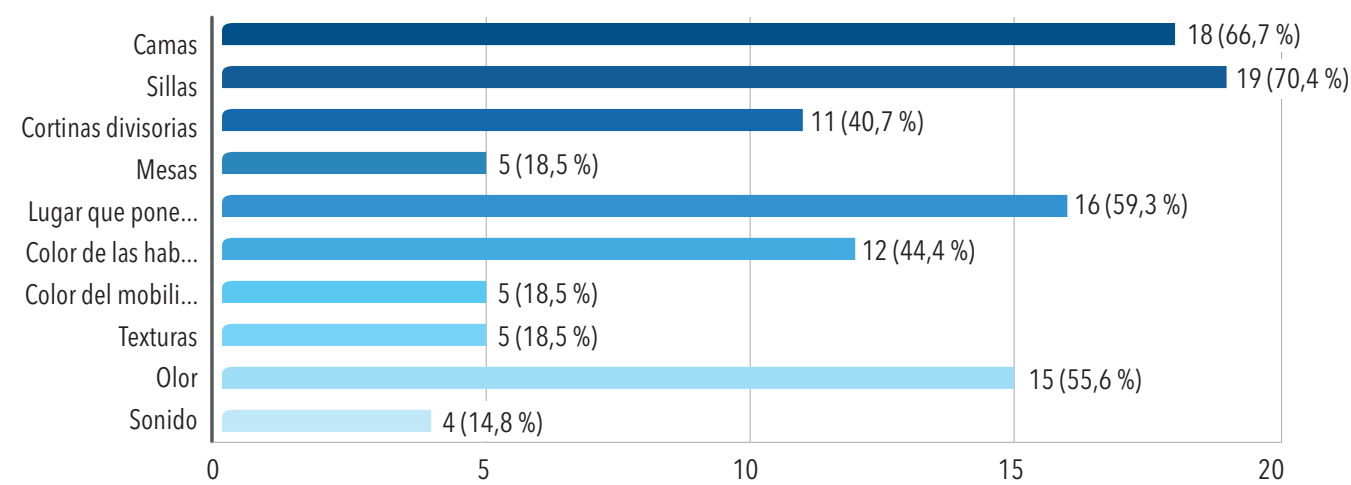

Gráfica 2. Percepción de posibles cambios. (Leija Macías, 2018). 
3. Los usuarios indirectos desean sentir el y de cabeza provocados por estar sentado en una simenor cansancio posible y evitar dolores musculares lla incómoda (Gráficas 3 y 4).

Pregunta 3: ¿Cómo se sintió físicamente durante este tiempo? (Puede seleccionar varias respuestas)

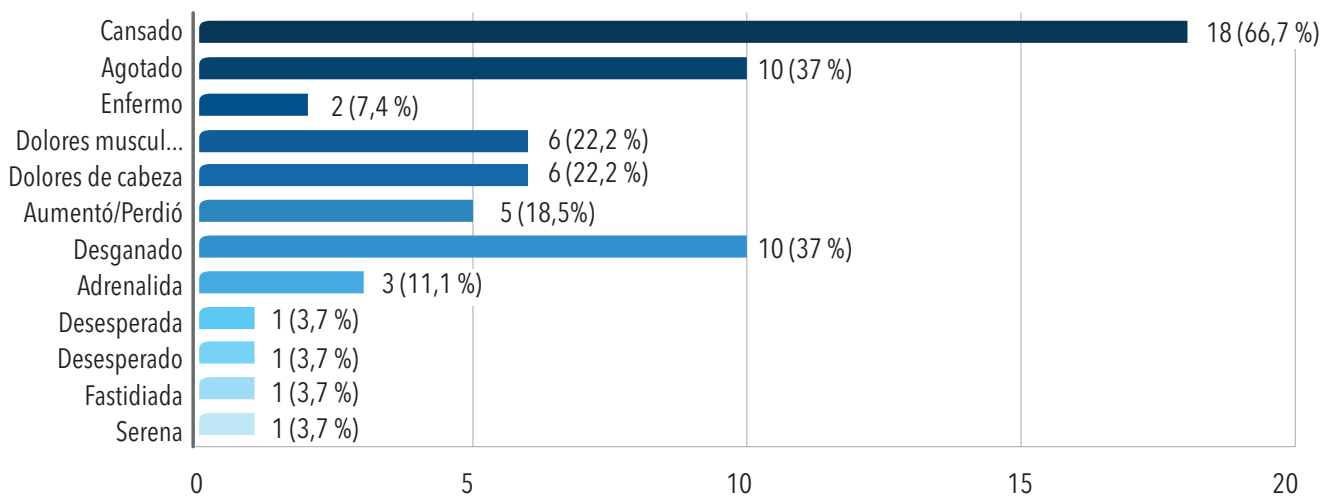

Gráfica 3. Descripción del sentir físico durante la estadía. (Leija Macías, 2018).

Pregunta 4: ¿Qué le gustaría que estos cambios transmitieran? (Seleccionar varias opciones)

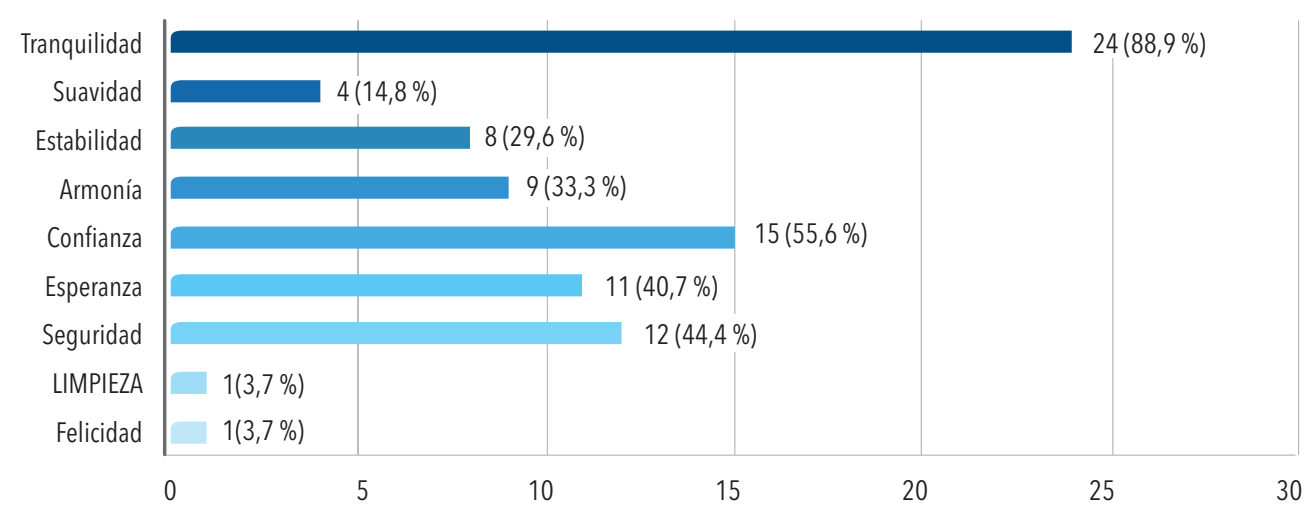

Gráfica 4. Emoción a transmitir por parte del mobiliario. (Leija Macías, 2018). 


\section{Conceptualización del diseño}

Después de detectar las principales problemáticas, y elegir con cuál se trabajaría gracias a la información obtenida del marco teórico y de la investigación de campo. Se puede dar una alternativa para solucionar la problemática de la incomodidad de las sillas donde las personas que cuidan a pacientes en hospitales pasan horas sentadas. Así, tomando en cuenta las necesidades detectadas (resultado de la investigación de campo elaborada previamente), se pueden considerar elementos clave para cumplir con el propósito de resolver las problemáticas a las que los usuarios se enfrentan durante su estadía en los hospitales públicos.

\section{Premisas}

En base a las necesidades detectadas, se diseñaron propuestas que cumplan con los siguientes requerimientos:
- Brindar una experiencia más favorable al usuario durante su estadía, proponiendo el uso de materiales cómodos con ángulos y medidas adecuadas para tener un mejor descanso.

- El diseño deberá contener un espacio para colocar las pertenencias, tanto del paciente como de su cuidador reduciendo así su preocupación por el cuidado de estas.

- En cuanto a su tamaño, se considerará respetar el espacio asignado a su lugar, intentando no estorbar el paso del personal de enfermería. - El mobiliario propuesto tendrá que ser agradable, cómodo y liviano.

- Debe ser intuitivo y fácil de utilizar.

\section{Proceso creativo y generación de propuesta final}

Con base a la investigación exploratoria y descriptiva, así como el uso de las diferentes herramientas de diseño, se realizó un proceso creativo para encontrar la mejor solución posible al problema. 

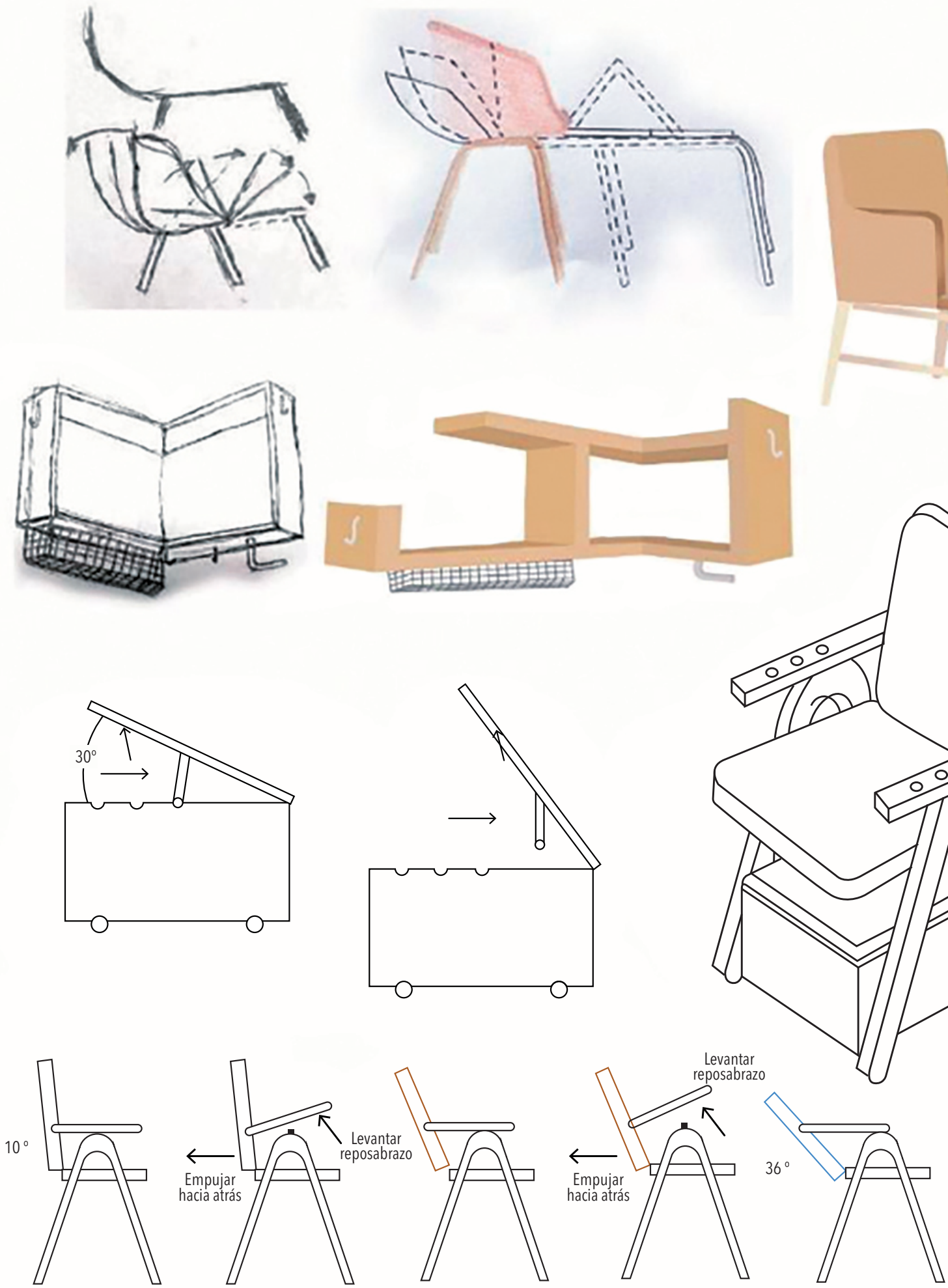

Figura 2. Proceso creativo: generación de bocetos y primeras ideas. (Leija Macías, 2018). 


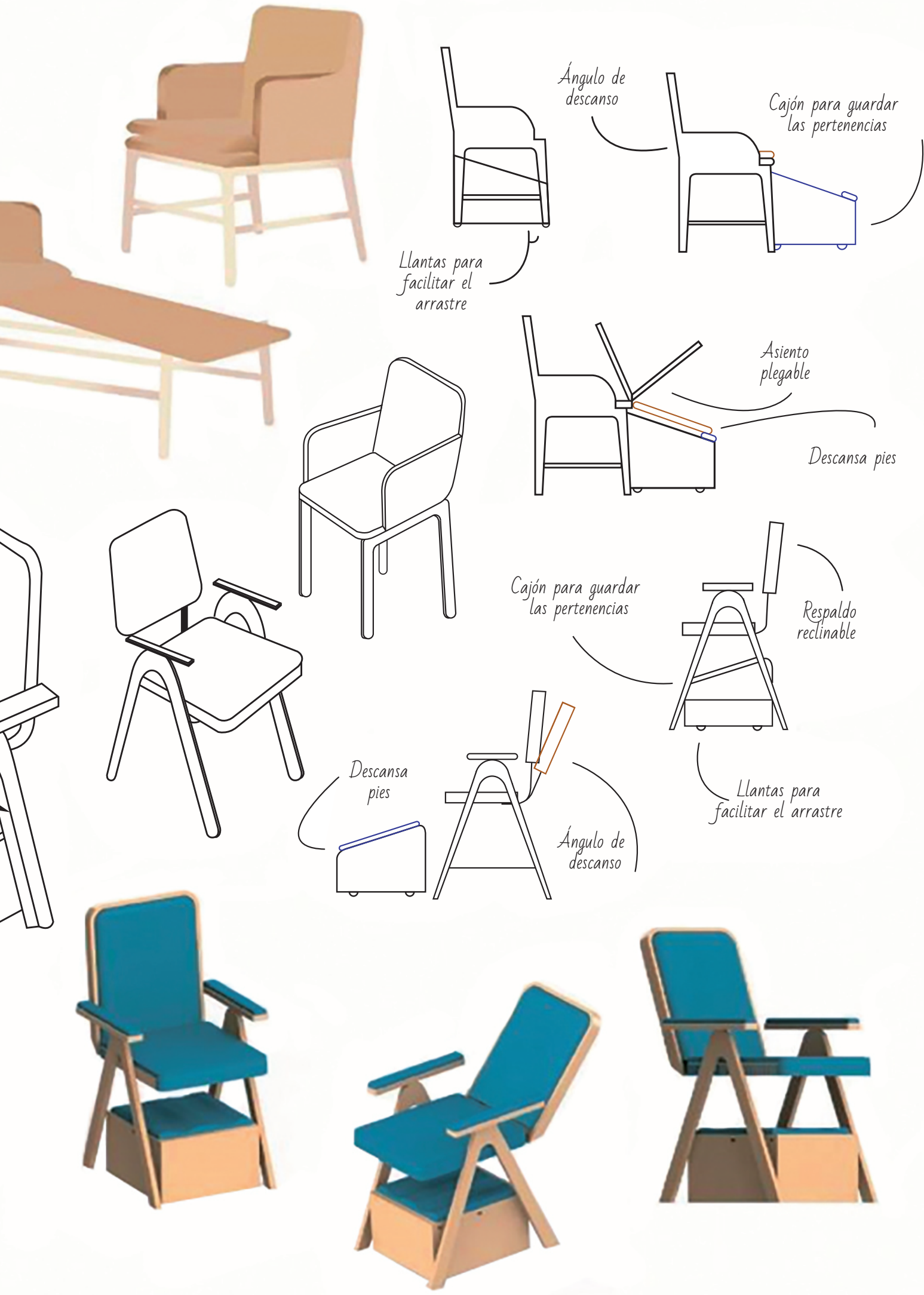




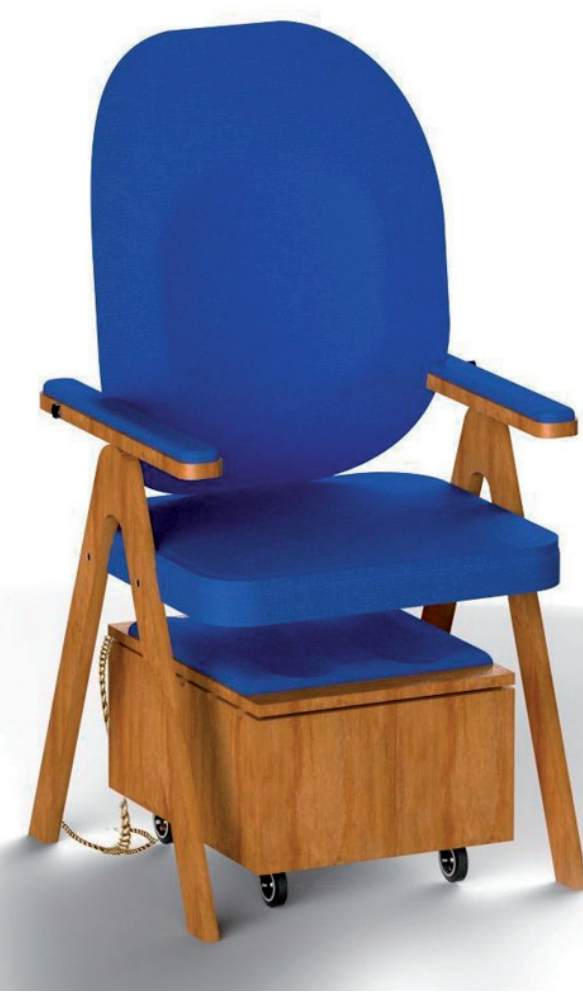

Figura 3. Propuesta final: Fidelity. (Leija Macías, 2018).

Finalmente, se llegó a la generación de esta propuesta: Fidelity (véase Figura 3). Donde el principal objetivo es mejorar la experiencia del usuario, el cual cuenta con tres ángulos distintos de inclinación en el respaldo, además de contener un cajón en la parte inferior para que en este pueda mantener almacenadas las pertenencias tanto del usuario directo como del indirecto (véase Figura 4). El mecanismo para realizar las inclinaciones se activa de manera manual mediante el descansa-brazo (véase Figura 5). 


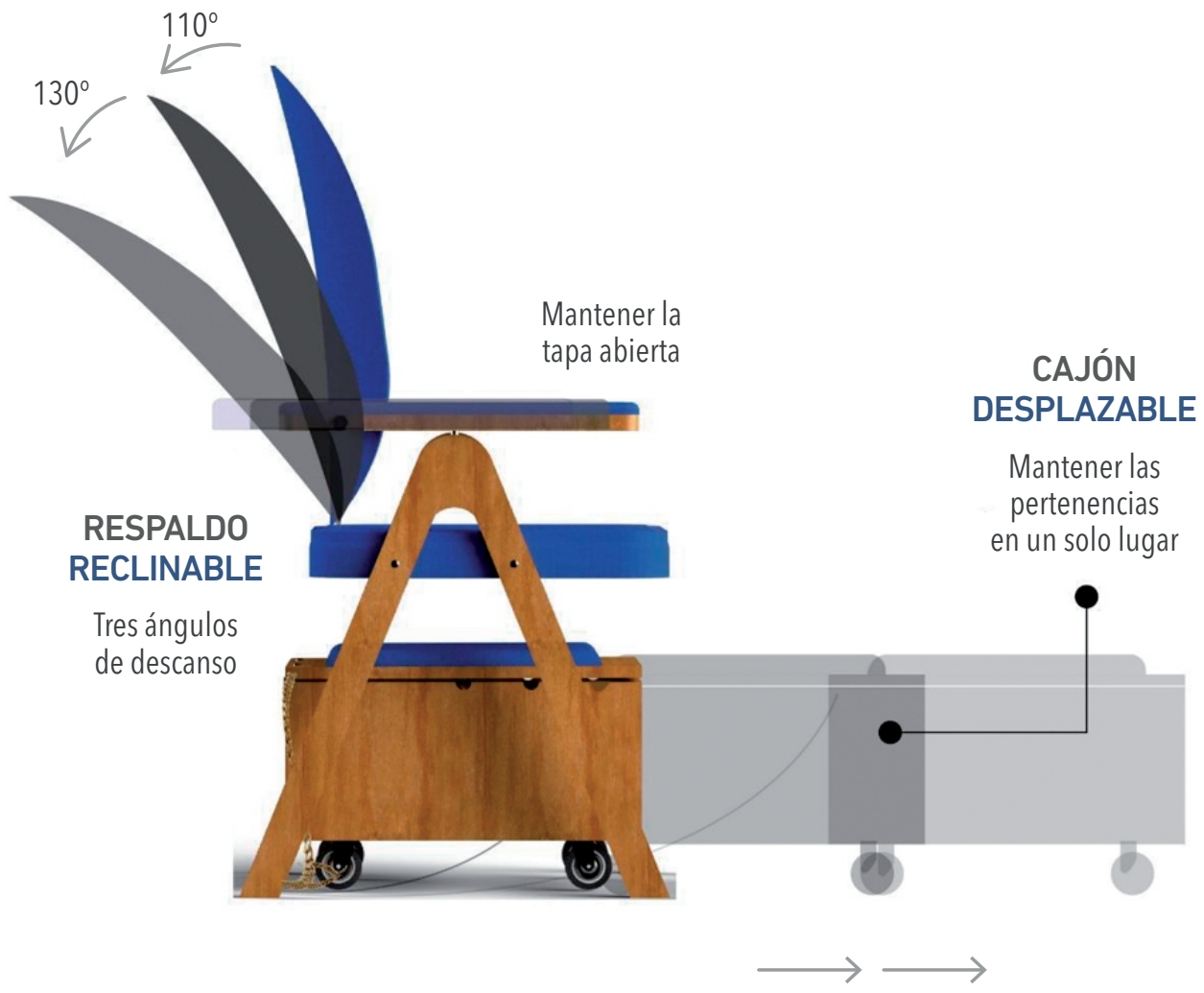

Figura 4. Detalle ángulos de inclinación y movimiento del cajón. (Leija Macías, 2018).

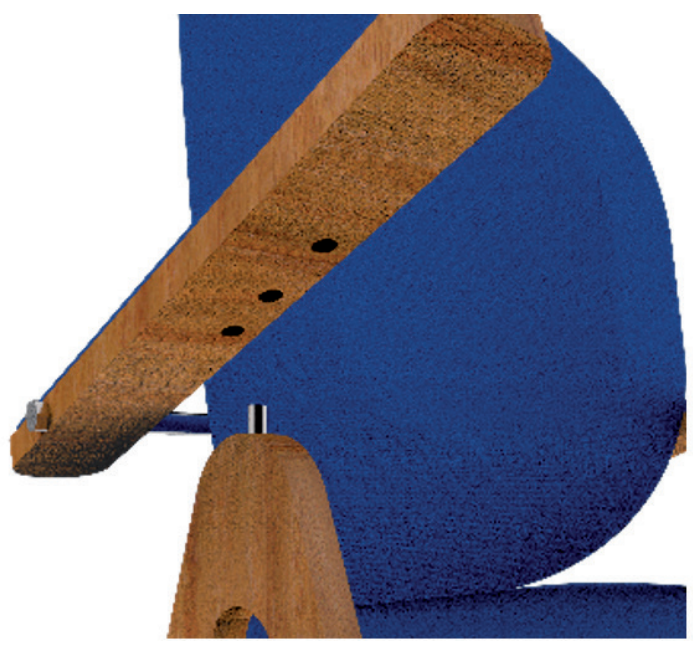

Figura 5. Detalle mecanismo manual. (Leija Macías, 2018). 
Realizado el análisis de las medidas antropométricas y los percentiles de México, se consideraron las medias de ancho, largo, alto, tamaño de descansa-brazo, ángulo de inclinación en los respaldos, entre otras, y de igual manera, las medidas del entorno donde se situará el objeto.

Dentro de los materiales que se propusieron para la elaboración, se encuentra el uso de MDF de pino para la estructura, memory foam como parte del relleno del respaldo. Además de aprovechar sus

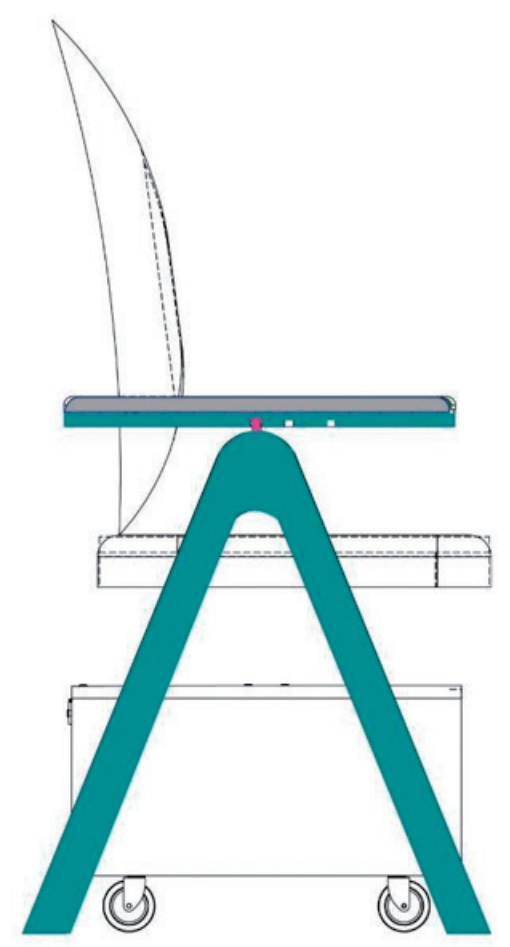

El cajón de las pertenencias se puede utilizar para que el usuario pueda estirar sus piernas encima de él, proporcionando así un mejor descanso (véase Figura 7). Como elemento adicional, cuenta con una pequeña cadena para mantener unido el características debido a que distribuye el peso sobre la superficie y ayuda a recuperar al cuerpo del estrés y la tensión, eliminando los puntos de presión, reduciendo el dolor, y manteniendo en la columna su alineación natural. Como forro se propone el uso de poliéster FR, el cual es fabricado con hilos hipo alergénicos que se puede desinfectar, no afecta la humedad, es ignífugo y especial para usar en zonas con mucho tránsito de personas (véase Figura 6).

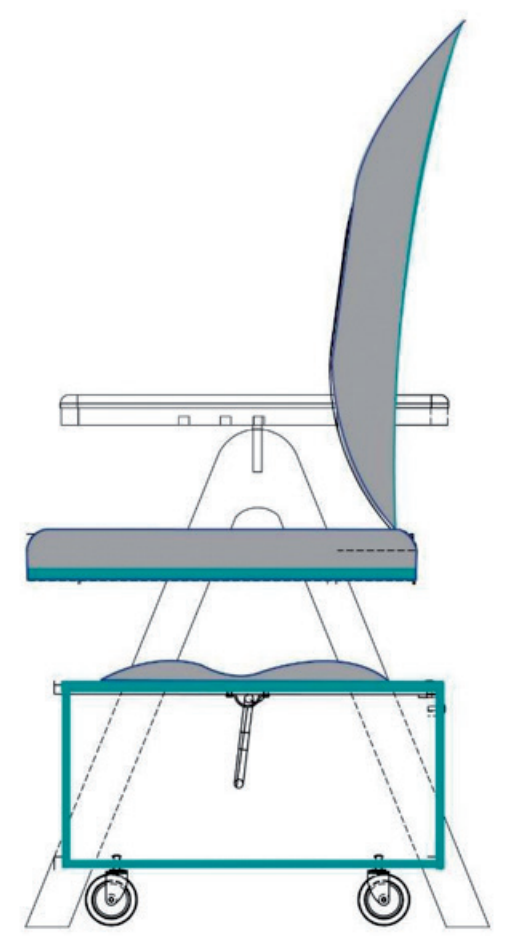

Figura 6. Vista de sección representando los materiales a utilizar. (Leija Macías, 2018). 


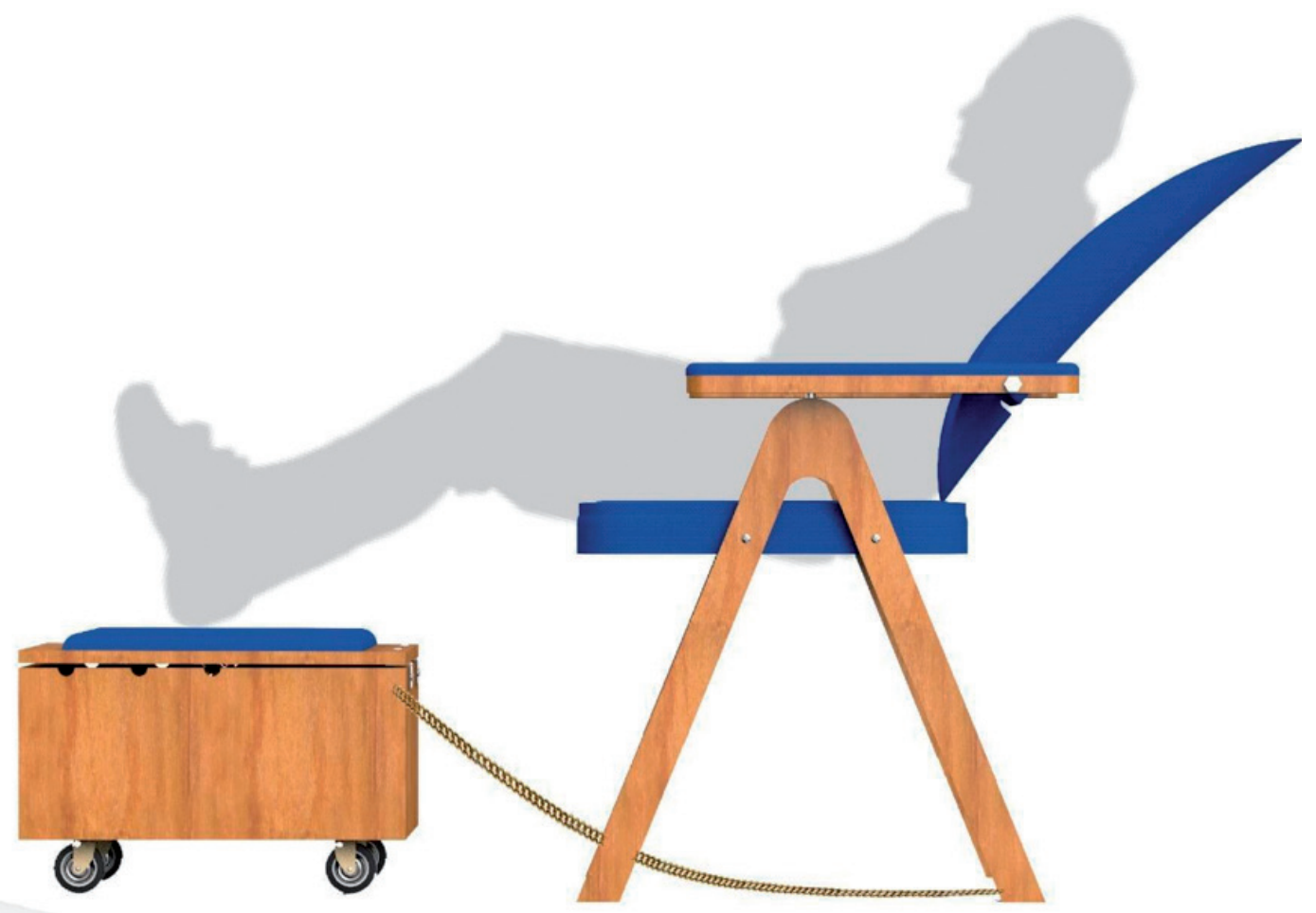

Figura 7. Representación de usuario utilizando el reposapiés. (Leija Macías, 2018).

Concluyendo con la vista frontal, superior y lateral de las medidas generales para una mejor representación del producto en su estado normal cuando el usuario (véase Figura 8), y de igual manera, la vista frontal, superior y lateral con las medidas cuando la persona está reclinada y utilizando el reposapiés (véase Figura 9). 


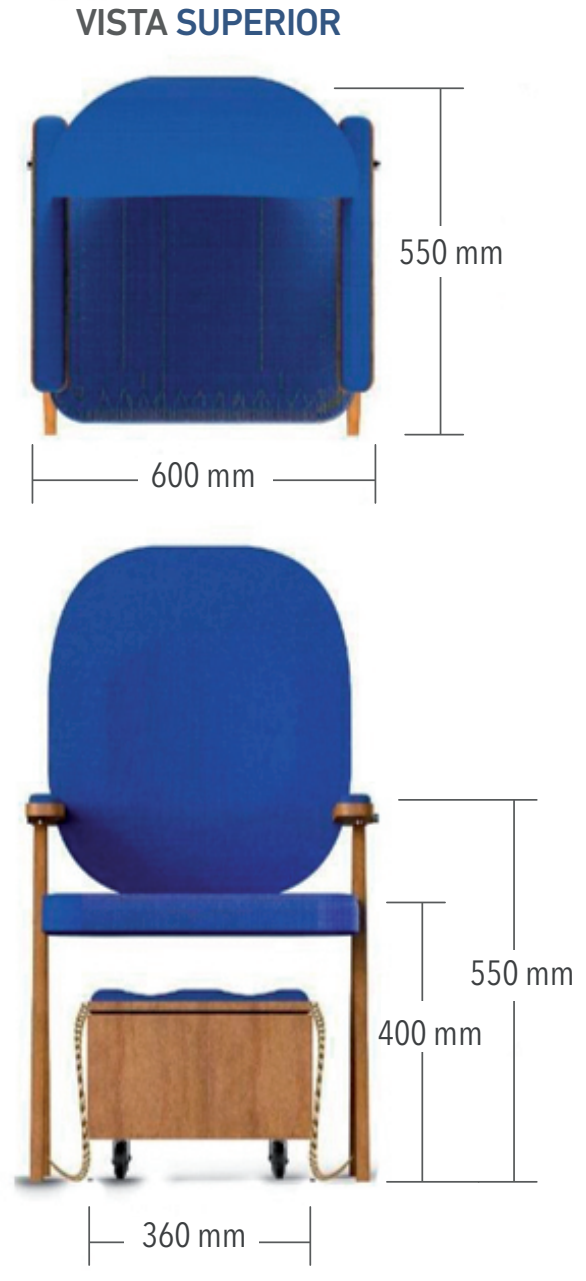

VISTA FRONTAL

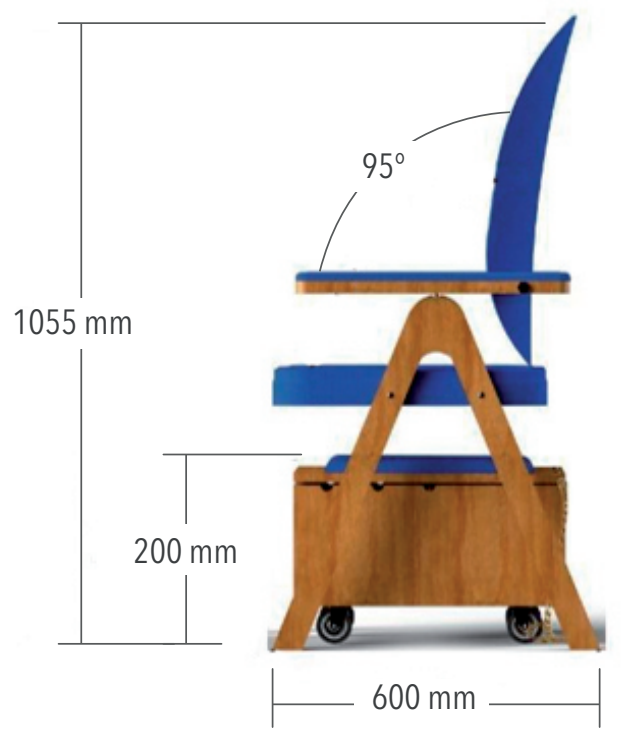

VISTA LATERAL

Figura 8. Vistas y medidas de la silla Fidelity. (Leija Macías, 2018). 


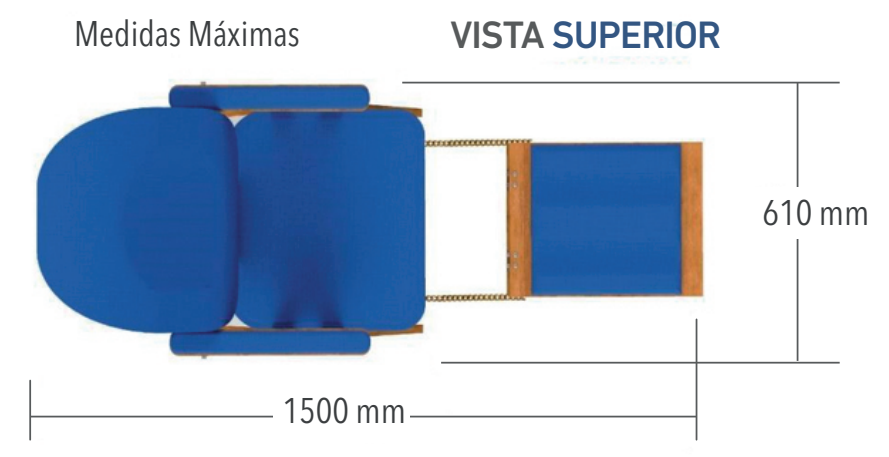

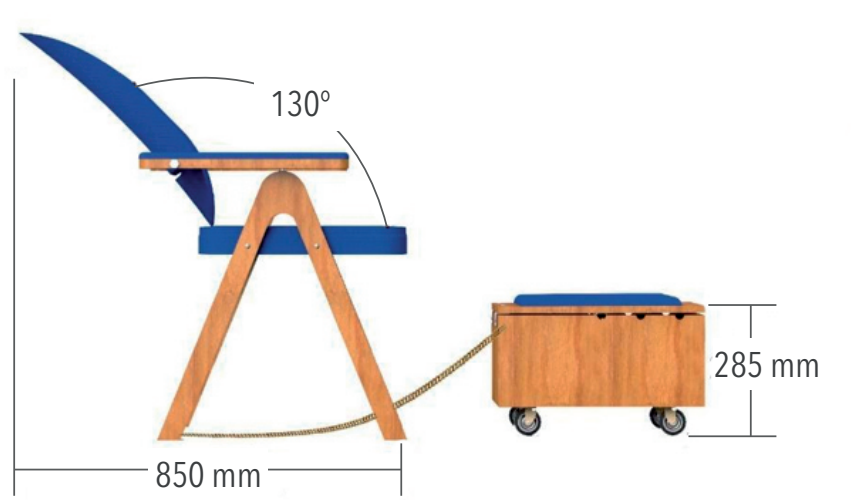

VISTA FRONTAL

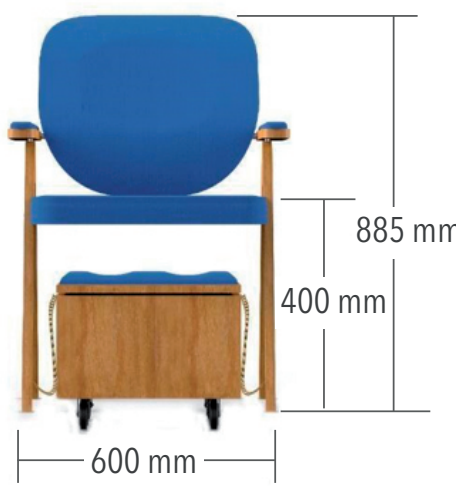

VISTA LATERAL

Figura 9. Vistas y medidas con reposapiés extraído. (Leija Macías, 2018).

\section{Conclusión}

Como conclusión, es evidente y clara la necesidad inherente de mejorar las condiciones de estancia de usuarios indirectos en los hospitales públicos de México. Son situaciones mentales muy demandantes para los usuarios, por lo tanto, es importante considerar la mejora sustancial de la experiencia al utilizar, productos y servicios en las clínicas. Como se mencionó anteriormente, el desgaste físico, desgaste emocional, impotencia, así como momentos de malestar en general, son situaciones que se viven a diario en los usuarios indirectos de los hospitales públicos.

Es importante comentar que todo lo que abarca el entorno de un hospital público no se puede cambiar de la noche a la mañana, y tampoco la gente va a cambiar su sentir y emociones que este lugar le genera, sin embargo, se puede ayudar a hacer su estancia menos estresante o cansada, siempre y cuando el producto esté bien pensado y diseñado para generar la experiencia y emociones deseadas. Como eje rector de un profesionista del diseño industrial, siempre se buscará la mejora continua en el estilo y calidad de vida de los seres humanos. 


\section{Referencias bibliográficas}

Agencia Reforma. (2010). El Siglo de torreón. Obtenido de https://www.elsiglodetorreon.com.mx/ noticia/518593.lideran-en-carencias-hospitales-mexicanos.html

Arhippainen, L. T. (2003). "Empirical Evaluation of User Experience in Two Adaptative Mobile Application Prototypes". 2nd International Conference on Mobile and Ubiquitous Multimedia. Norrköping, Sweden. Obtenido de http://www.ep.liu.se/ecp/011/007/ecp011007.pdf

Bedolla, D. (2004). Diseño sensorial: modelos guia para la concepción de. Obtenido de http://cmap. upb.edu.co/servlet/SBReadResourceServlet?rid=1153176144421_693802693_1561

Casillas, L. P. (2017). Valor emocional en los productos. Obtenido de https://medium.com/@luceropinedocasillas/valor-emocional-en-los-productos-4f2572813e0a

Crossley, L. (2003). "Building emotions in design". The Design Journal, 6(3), pp. 35-45. Obtenido de https://www.tandfonline.com/doi/abs/10.2752/146069203789355264

Garcia del Arco, J. (2014). Innovación desde Experiencia Cliente. Obtenido de https://mba. americaeconomia.com/sites/mba.americaeconomia.com/files/xuperamartesinnobasquev01-140328111348-phpapp01.pdf

IMSS. (2017). Arranca recaudación del programa Sillas-Cama 2017. Obtenido de http://www.imss. gob.mx/prensa/archivo/201704/123

IMSS. (2017). Directorio de instalaciones del IMSS. Obtenido de http://www.imss.gob.mx/directorio/

Instituto de Seguridad Social del Estado de México y Municipios. (2017). Hospitalización. Obtenido de http://www.issemym.gob.mx/tu_salud/hospitalizaci\%C3\%B3n

Knapp Bjerén, A. (2003). La Experiencia del Usuario. Madrid: Anaya Multimedia.

Nella, J. I. (2014). Diseño emocional y experiencia de usuario. Obtenido de http://sedici.unlp.edu. ar/bitstream/handle/10915/62697/Documento_completo.pdf-PDFA.pdf?sequence=1\&isAllowed =y

Norman, D.A. (2004). Emotional Design; why we love (or hate) everyday things. EE.UU.: Basic Books.

Real Academia Española. (2017). Emoción. Obtenido de http://dle.rae.es/?id=EjXPOmU

Real Academia Española. (2014). Experiencia. Obtenido de http://dle.rae.es/?id=HlelZIn

Revista M\&M. (2016). "El diseño emocional como valor agregado en el mobiliario" Revista M\&M. Obtenido de https://revista-mm.com/blog/secciones-tema/diseno/diseno-emocional-agregado-mobiliario/

Shedroff, N. (2001). Experience Design. EE.UU: New Riders Publishing.

Subdirección de Atención Integral al Paciente. (s.f). Reglamento e información para familiares de pacientes hospitalizados. Obtenido de http://himfg.com.mx/descargas/documentos/transparencia/tripticos/reglamento.pdf 


\section{Anexo 1}

\section{ENCUESTA ELECTRÓNICA}

\section{Hospitales + Diseño Emocional}

¿De qué manera el diseño emocional puede ayudar a mejorar la estadía de los pacientes y sus familiares durante el proceso de recuperación en hospitales públicos en México?

1. Edad:

2. Género

Mujer $\bigcirc$ Hombre $\bigcirc$ Otro

3. Tipo de Hospital en el que ha tenido ésta experiencia:

Público Privado

4. El papel principal que tenía durante esta fue como:

$\bigcirc$ Paciente $\bigcirc$ Cuidador Familiar $\bigcirc$ Cuidador $\bigcirc$ Médico, Enfermero, Personal $\bigcirc$ Visitante

5. Además de ser cuidador/paciente, ¿También ha estado internado/sido cuidador?:

Sí. En un hospital público. $\bigcirc$ Sí. En un hospital privado. $\bigcirc$ No.

6. ¿Cuál fue la diferencia entre ambas experiencias? (Si su respuesta anterior fue "no", responda aquí "no aplica"):

7. Previamente eligió el papel principal que tenía, ¿Cuál fue el tiempo de estadía durante esta experiencia?

Menos de 7 días $\bigcirc$ entre 7 y 15 días $\bigcirc$ Entre 16 días y un mes
Entre 1 y 2 meses $\bigcirc$ 0tros:

8. Su experiencia en el Hospital fue...

$\bigcirc$ Muybuena $\bigcirc$ Buena $\bigcirc$ Regular $\bigcirc$ Mala $\bigcirc$ Muy Mala

9. ¿Cómo era el entorno que lo rodeaba? (Puede seleccionar más de una opción)

$\begin{array}{llll}\text { Cálido } & \text { Frío } & \text { Deprimente } & \text { Sucio } \\ \text { Limpio } & \text { Divertido } & \text { Calmado } & \text { Inquietante } \\ \text { Aburrido } & \text { Feo } & \text { Permentoso } & \text { Incómodo } \\ \text { Amigable } & \text { Intros: } & & \end{array}$

10. ¿Considera que éste entorno influyó en su estado de ánimo?:

$\bigcirc$ Sí $\bigcirc$ No 
11. ¿Cómo se sintió físicamente durante este tiempo? (Puede seleccionar varias respuestas)

$\begin{array}{lll}\text { Cansado } & \text { Agotado } & \text { Onfermo } \\ \text { Adrenalina } & \text { Dolores de cabeza } & \text { Pumentó/Perdió peso } \\ \text { Desganado } & \text { Dolores Musculares } & \text { Otros: }\end{array}$

12. ¿Qué emociones le transmite estar en un Hospital Público? (Puede seleccionar más de una opción)
Felicidad
Estrés
Inquietud
Otros
Angustia
Tranquilidad
Temor
Confianza
Desesperación
Ansiedad
Tristeza
Seguridad

13. La habitación en la que se encontraba, ¿era individual o compartida?:
Individual
Compartida

14. Describa brevemente, ¿Cómo era ésta habitación?:

15. ¿De qué manera pasaba el tiempo durante su estadía? (Seleccione al menos una)

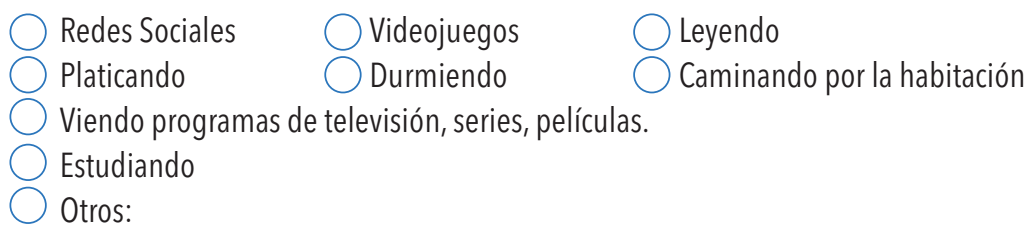

16. Del 1 al 5 , ¿Qué tanto cree que el Diseño del lugar y de los objetos influyen en su estado de ánimo? (5 es

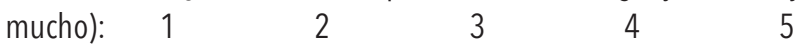

17. ¿Cómo le gustaría que fuera el mobiliario utilizado en las habitaciones?

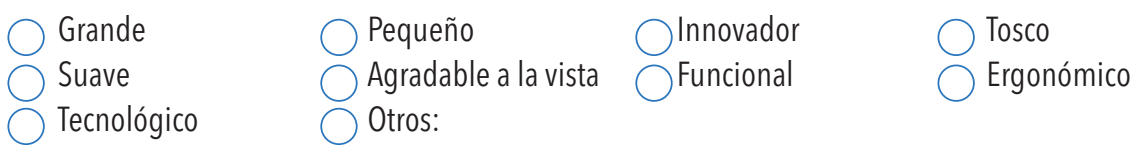

18. Si tuviera la oportunidad de modificar algo dentro de los cuartos de recuperación, ¿Qué sería? (Puede seleccionar diversas opciones)

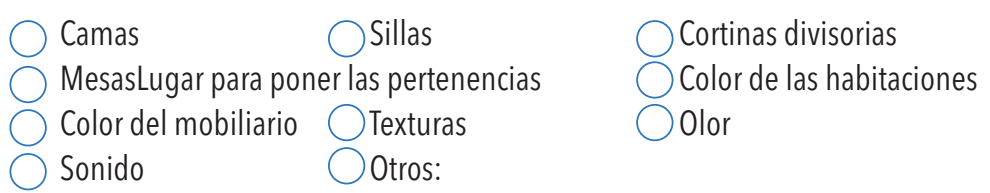


19. ¿Qué le gustaría que estos cambios transmitieran? (Seleccionar varias opciones)

$\begin{array}{lll}\text { Tranquilidad } & \bigcirc \text { Suavidad } & \text { Estabilidad } \\ \text { Armonía } & \bigcirc \text { Confianza } & \text { Ssperanza } \\ \text { Seguridad } & \bigcirc \text { Otros: } & \end{array}$

20. Si fuera tratado en un hospital privado por el mismo equipo médico y de enfermería, ¿Cree que su experiencia seria la misma? Explique su respuesta:

\section{VISITANTE}

1. ¿Cuál fue la primera impresión al entrar y ver la habitación?

\begin{tabular}{|c|}
\hline$\left\{\begin{array}{l}\text { Desagradable } \\
\text { Limpia } \\
\text { Otros: }\end{array}\right.$ \\
\hline
\end{tabular}

2. ¿Qué emociones le transmitía estar ahí? (Puede seleccionar más de una opción)
Tristeza
Preocupación
Alivio
Alegría
Miedo
Esperanza
Tranquilidad
Confort
Seguridad
Desconfianza
Incomodidad
Otros:

3. Describa breve mente qué modificaría de las habitaciones: 
